\title{
Predicting Factors for High-Grade Cervical Dysplasia in Women With Low-Grade Cervical Cytology and Nonvisible Squamocolumnar Junction
}

Reproductive Sciences I-5

(C) The Author(s) 2018 Reprints and permission: sagepub.com/journalsPermissions.nav DOI: $10.1177 / 19337|9| 18756747$ journals.sagepub.com/home/rsx @SAGE

\author{
Giorgio Bogani, MD, PhD', Francesca Taverna, MD², \\ Claudia Lombardo, MD², Antonino Ditto, MD', \\ Fabio Martinelli, MD'D, Mauro Signorelli, MD', \\ Valentina Chiappa, MD', Umberto Leone Roberti Maggiore, MD', \\ Lavinia Mosca, MD', Ilaria Sabatucci, MD', Cono Scaffa, MD, PhD', \\ Domenica Lorusso, MD, PhD', and Francesco Raspagliesi, MD'
}

\begin{abstract}
Objective: To assess the risk of developing high-grade cervical dysplasia among women with low-grade cervical cytology and nonvisible squamocolumnar junction (SCJ) at colposcopic examination. Methods: Data of consecutive women with lowgrade intraepithelial lesion $(\leq \mathrm{LSIL})$ undergoing colposcopic examination, which was unsatisfactory (due to the lack of the visualization of the entire SCJ), were retrospectively reviewed. The risk of developing high-grade cervical intraepithelial neoplasia (CIN2+) was assessed using Kaplan-Meier and Cox models. Results: Data of 86 women were retrieved. Mean (standard deviation [SD]) age was 36.3 (I3.4) years. A total of $7 \mathrm{I}$ (82.5\%) patients had high-risk human papillomavirus (HR-HPV) at the time of diagnosis. Among the 63 patients undergoing repetition of HPV testing, $15(24 \%)$ and 48 (76\%) women had positive and negative tests for HR-HPV at 12 months, respectively. We observed that $5(33 \%)$ of 15 patients with HPV persistence developed CIN2+, while only I (2\%) patient of 48 patients without HPV persistence developed CIN2+ (odds ratio [OR]: 23.5; $95 \%$ confidence interval $[\mathrm{Cl}]$ : 2.46-223.7; $P<.00 \mathrm{I})$. The length of HR-HPV persistence correlated with an increased risk of developing $\mathrm{CIN} 2+(P<.001$; $P$ for trend). High-risk HPV persistence is the only factor predicting for $\mathrm{CIN} 2+$ (hazard ratio: 3.19 ; $95 \%$ CI: $1.55-$ 6.57; $P=.002$ ). Conclusions: High-risk HPV persistence predicts the risk of developing CIN2 + in patients with unsatisfactory colposcopic examination. Further studies are warranted in order to implement the use of HPV testing in patients with unsatisfactory colposcopy.
\end{abstract}

\section{Keywords}

HPV, high-grade dysplasia, unsatisfactory colposcopy, CIN

\section{Introduction}

Colposcopy allows identification of abnormal areas, including low- and high-grade cervical intraepithelial neoplasia (CIN1 and $\mathrm{CIN} 2+$, respectively). ${ }^{1-3}$ Lesions are generally located in the transformational zone (TZ) close to the squamocolumnar junction (SCJ). ${ }^{4}$ The evaluation of TZ and SCJ is paramount to assess the status of the uterine cervix, in case of human papillomavirus (HPV) infection. Therefore, the visualization of the SCJ is essential to assess the quality of colposcopic examination.

According to the old nomenclature, the lack of the visualization of the entire SCJ defined a colposcopy as "unsatisfactory." Although the new nomenclature refused the term "unsatisfactory colposcopy," the not complete visualization of the SCJ is still a concern.
It is estimated that the entire SCJ is not visualized in about $10 \%$ to $20 \%$ of colposcopic examinations, thus making these evaluation less precise than in case in which the SCJ is fully visualized. Several investigators recommended cone biopsy in those cases. ${ }^{5,6}$

\footnotetext{
' Department of Gynecologic Oncology, IRCCS National Cancer Institute, Milan, Italy

2 Department of Immunohematology and Transfusion Medicine Service, IRCCS National Cancer Institute, Milan, Italy
}

Corresponding Author:

Giorgio Bogani, Department of Gynecologic Oncology, National Cancer Institute, Via Venezian I, 20I33 Milan, Italy.

Email: giorgiobogani@yahoo.it 


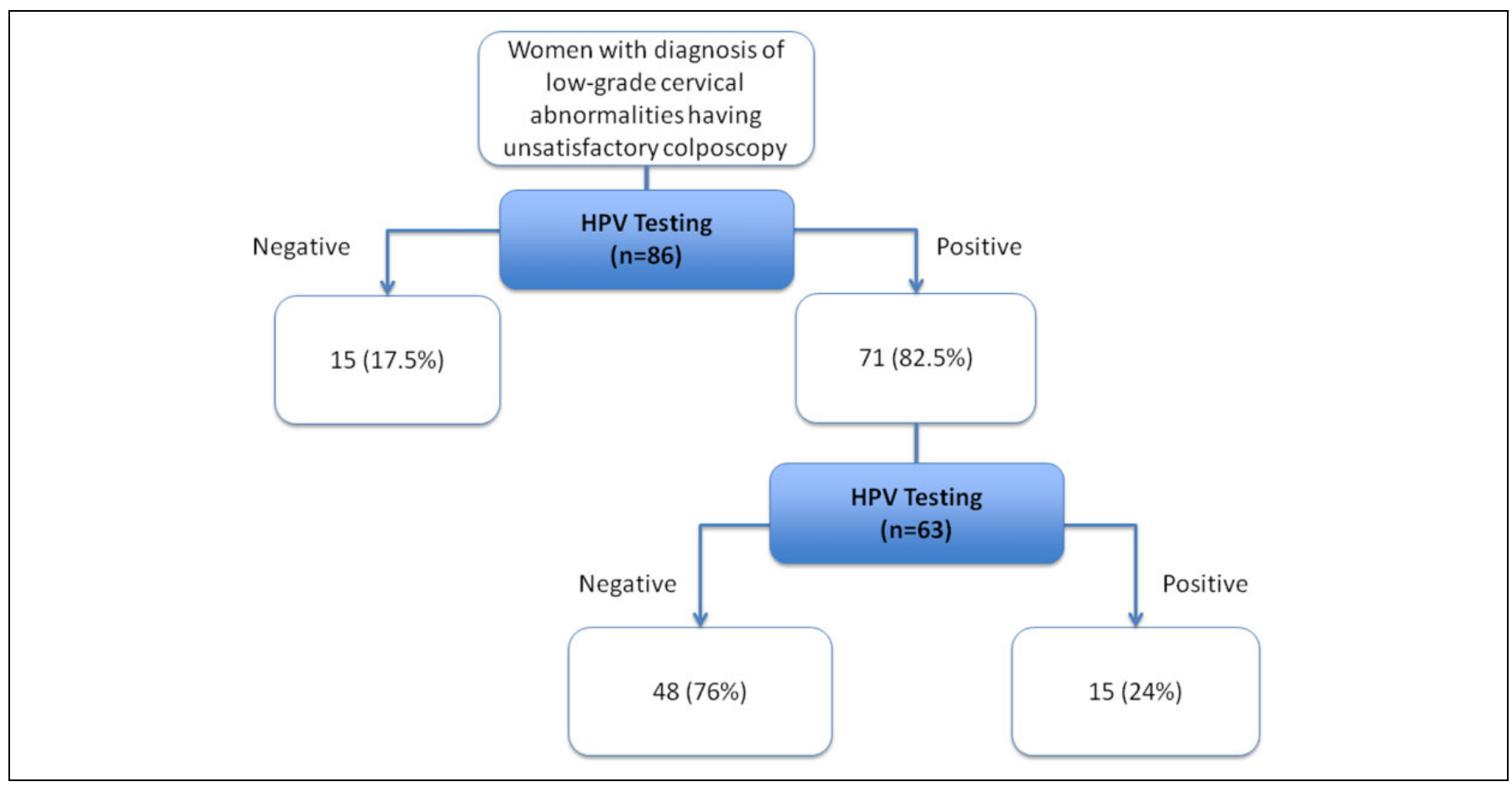

Figure I. Study design.

In the present study, we sought to assess the risk of developing high-grade lesions (CIN2+) among women with low-grade cervical cytology and unsatisfactory colposcopic examination. Additionally, we aimed to identify factors predicting CIN2+ among this cluster of women.

\section{Methods}

We retrospectively reviewed records of all consecutive women undergoing HPV DNA testing between 2005 and 2015 at Gynecologic Oncology Unit of National Cancer Institute (Milan, Italy). The institutional review board approved this study.

Data of all patients included were abstracted from a dedicated database including all women undergoing HPV testing. Data were included prospectively into the database. All patients included gave written informed consent for the use of personal information for health research. Demographic details, data about HPV type(s) detected, and data on treatment for the occurrence of genital precancerous and cancerous condition were retrospectively reviewed. Low-grade cervical cytology included low-grade intraepithelial lesion (LSIL) and atypical squamous cells of undetermined significance. Unsatisfactory colposcopy was defined according to the old nomenclature realized by the International Federation for Cervical Pathology and Colposcopy (IFCPC). ${ }^{4}$ Human papillomavirus types were considered as high risk (HR) according to the data of the International Agency for Research on Cancer. ${ }^{7}$ High-risk HPV types classified as type 1 carcinogens included HPV16, $18,31,33,35,39,45,51,52,56,58,59$, and 66 . Figure 1 shows the flow of patients into the study design.
Age was categorized as the follows: age less than 30 years, between 30 and 50 years, and more than 50 years. On the basis of body mass index (BMI), women were categorized into normal weight (BMI less than $25 \mathrm{~kg} / \mathrm{m}^{2}$ ), overweight (BMI more than 25 less than $30 \mathrm{~kg} / \mathrm{m}^{2}$ ), and obese (BMI more than $30 \mathrm{~kg} / \mathrm{m}^{2}$ ). Tobacco use was defined as a dichotomous variable (yes vs no). Exclusion criteria were as follows: (1) age younger than 18 years, (2) withdrawal of consent, (3) history of HPV-related lesions (including cervical, vaginal, and vulvar dysplasia related to HPV infections) within the past 5 years, (4) pregnancy, and (5) previous hysterectomy. According to our institutional protocol, patients were evaluated colposcopically in outpatient clinic at 6 months after primary colposcopic examination. A dedicated team of gynecologic oncologists performed all gynecological and colposcopic examinations. Details regarding colposcopic examination, HPV testing, and HPV analysis are reported elsewhere. ${ }^{8}$ The genotyping results were ranked hierarchically according to cancer risk: HPV $16>$ HPV $18>$ other carcinogenic HPV. Generally, HPV testing was performed at the time of first colposcopic examination. In our department, no specific guidelines suggested the repetition of HPV testing. However, the majority of women with the diagnosis of HR-HPV had another HPV testing at 6 or 12 months after primary examination. ${ }^{8}$ Human papillomavirus infection persistence was defined as the persistence of an HPV type, detected at 2 consecutive time points, in accordance with the definition included in the meta-analysis of Koshiol et al. ${ }^{9}$

Data are summarized using basic descriptive statistics. The risk of developing CIN2+ was evaluated using Kaplan-Meir and Cox models. Hazard ratio and $95 \%$ confidence intervals (CIs) were 
Table I. Baseline Patient Characteristics.

\begin{tabular}{lc}
\hline Characteristics & $\mathrm{N}=86$ \\
\hline Age, median (IQR), years & $32.5(26,45.2)$ \\
BMI, median (IQR), kg/m² & $22(21,24.2)$ \\
Tobacco use, $\mathrm{n}(\%)$ & $68(79 \%)$ \\
$\quad$ No & $18(21 \%)$ \\
$\quad$ Yes & $71(82.5 \%)$ \\
Positivity for HPV at diagnosis, $\mathrm{n}(\%)$ & $37(43 \%)$ \\
Positivity for HPV I6/I8 at diagnosis, $\mathrm{n}(\%)$ & $34(39.5 \%)$ \\
Positivity for HPV other than I6/I8 at diagnosis, $\mathrm{n}(\%)$ & $63(73.2 \%)$ \\
Patients undergoing another HPV testing after & \\
$\quad$ primary examination & $\mathrm{I} 5(17.4 \%)$ \\
Persistence of HPV, $\mathrm{n}(\%)$ & $6(6-18)$ \\
Time of HPV persistence, median (IQR), months & $29(23-60)$ \\
Follow-up, median (IQR), months &
\end{tabular}

Abbreviations: BMI, body mass index; HPV, human papillomavirus; IQR, interquartile range; SD, standard deviation.

calculated for each comparison. Univariate and multivariate analyses were performed when appropriate. All covariates with a $P$ value less than .10 , based on univariate analysis, were included in the multivariate model. Duration of follow-up was counted from date of LSIL detection and of CIN2+ diagnosis or date of last follow-up. Statistical analyses were performed using GraphPad Prism version 6.0 for Mac (GraphPad Software, San Diego, California) and IBM-Microsoft SPSS (SPSS Statistics. International Business Machines Corporation IBM. 2013. Armonk) version 20.0 for Mac.

\section{Results}

Data of 86 women diagnosed with low-grade cervical cytology were retrieved. Table 1 reports baseline characteristics of the study population. Mean (standard deviation [SD]) age was 36.5 (12.1) years. Seventy-one (82.5\%) patients had HR-HPV at the time of diagnosis. Among the 63 patients undergoing repetition of HPV testing, 15 (24\%) and 48 (76\%) women had positive and negative tests for HR-HPV at 12 months, respectively. After a median follow-up of 29 (75\% interquartile range: $23-$ $60)$ months, $8(9.3 \%)$ patients had conization due to CIN2+ (data not shown). No invasive cancer was diagnosed.

Table 2 reports factors predicting the risk of developing $\mathrm{CIN} 2+$. Considering factors predicting the risk of developing CIN2+ over time (median follow-up: 29 months), HR-HPV persistence correlated with the risk of developing CIN2+ (hazard ratio: $3.19 ; 95 \%$ CI: $1.55-6.57 ; P=.002$ ). Similarly, length of HR-HPV persistence correlated with an increased risk of developing $\mathrm{CIN} 2+(P<.001 ; P$ for trend - data not shown $)$. No other factors correlated with the risk of developing CIN2+. However, although it did not reach statistical significance, we observed that women with overweight (BMI between 25 and 30 $\mathrm{kg} / \mathrm{m}^{2}$ ) experienced a higher risk of developing CIN2 + (hazard ratio: 2.72 ; $95 \% \mathrm{CI}: 0.54-13.6 ; P=.22$ ).

Looking at the crude number of CIN2 + diagnosed, we observed that $5(33 \%)$ of 15 patients with HPV persistence at
Table 2. Factors Influencing the Risk of Developing CIN2+. ${ }^{a}$

\begin{tabular}{|c|c|c|c|c|}
\hline Characteristics & $\begin{array}{l}\text { Number } \\
\text { of } \\
\text { Patients }\end{array}$ & $\begin{array}{l}\text { Number } \\
\text { of } \\
\text { CIN2+ }\end{array}$ & $\begin{array}{l}\text { Univariate } \\
\text { Analysis, HR } \\
(95 \% \mathrm{Cl})\end{array}$ & $\begin{array}{c}P \\
\text { Value }\end{array}$ \\
\hline \multicolumn{5}{|l|}{ Age, years } \\
\hline Less than 30 years & 33 & $4(12.1 \%)$ & $1.54(0.38-6.19)$ & .53 \\
\hline $\begin{array}{l}\text { Between } 30 \text { and } \\
50 \text { years }\end{array}$ & 39 & $2(5.1 \%)$ & $0.42(0.08-2.09)$ & .29 \\
\hline $\begin{array}{l}\text { More than } 50 \text { years } \\
\mathrm{BMI}, \mathrm{kg} / \mathrm{m}^{2}\end{array}$ & 14 & $2(14.3 \%)$ & $1.66(0.33-8.23)$ & .53 \\
\hline Normal weight & 54 & $4(7.4 \%)$ & $0.78(0.24-5.59)$ & .12 \\
\hline Overweight & 20 & $3(15 \%)$ & $2.72(0.54-13.6)$ & .22 \\
\hline Obese & 13 & I (7.7\%) & $0.04(0.00-488.3)$ & .50 \\
\hline Tobacco use & & & & .52 \\
\hline No & 68 & 7 (10.3\%) & Reference & \\
\hline Yes & 18 & I (5.5\%) & $0.50(0.06-4.08)$ & \\
\hline $\begin{array}{l}\text { Positivity for HR-HPV } \\
\text { at diagnosis }\end{array}$ & & & & .79 \\
\hline No & 15 & I (6.7\%) & Reference & \\
\hline Yes & 71 & $7(9.8 \%)$ & $1.32(0.16-10.8)$ & \\
\hline $\begin{array}{l}\text { Positivity for HPV } \\
16 / 18 \text { at diagnosis }\end{array}$ & & & & .76 \\
\hline No & 49 & $5(10.2 \%)$ & Reference & \\
\hline Yes & 37 & $3(8.1 \%)$ & $0.80(0.19-3.37)$ & \\
\hline $\begin{array}{l}\text { Positivity for HR-HPV } \\
\text { other than } 16 / 18 \text { at } \\
\text { diagnosis }\end{array}$ & & & & .73 \\
\hline No & 52 & $4(7.7 \%)$ & Reference & \\
\hline Yes & 34 & $4(\mathrm{I} \mid .7 \%)$ & $0.73(0.3 I-5 . I I)$ & \\
\hline HR-HPV persistence & & & & .002 \\
\hline No & 48 & I (2\%) & Reference & \\
\hline Yes & 15 & $5(33 \%)$ & $3.19(1.55-6.57)$ & \\
\hline
\end{tabular}

Abbreviations: $\mathrm{BMI}$, body mass index; $\mathrm{Cl}$, confidence interval; $\mathrm{CIN}$, cervical intraepithelial neoplasia; HPV, human papillomavirus; HR, hazard ratio; HR$\mathrm{HPV}$, high-risk human papillomavirus.

${ }^{a} V$ ariables characterized by $P$ value $<.10$ are presented in bold characters.

12 months developed CIN2+, while only $1(2 \%)$ of 48 patients without HPV persistence developed CIN2+ (odds ratio: 23.5; 95\% CI: 2.46-223.7; $P<.001$; Table 2). Type-specific HRHPV infection did not influence the risk of developing CIN2+. In fact, comparing women having an infection from HPV 16, HPV 18, and other HR-HPV types, we observed a similar risk at 10-year follow-up $(P=.89, \log$-rank test). Similarly, no differences were observed comparing HPV 16 and HPV 18 $(P=.61$, log-rank test $)$.

\section{Discussion}

The present study evaluated outcomes of women diagnosed with low-grade cervical cytology and characterized by an unsatisfactory colposcopic examination, thus reporting a number of noteworthy findings. First, we observed that women diagnosed with low-grade cervical cytology have a risk of about $30 \%$ of developing CIN2 + in case of positivity for HR-HPV, while in the absence of HR-HPV infection, this risk is negligible. Second, persistence of HR-HPV is the main factor predicting this risk. Third, type-specific HPV infection does not influence 
patient outcomes. Additionally, in our series, other baseline factors (such as age or BMI) do not impact on the risk of developing CIN2+.

In the present study, we analyzed a particular subset of patients for whom colposcopic examination was not able to define the entire SCJ. According to the nomenclature introduced in 2002, these colposcopic examinations are defined as "unsatisfactory." On the other hand, colposcopy was defined "satisfactory" in case of complete visualization of the SCJ. In 2011, the new nomenclature realized by the IFCPC replaced the terms "satisfactory colposcopy" and "unsatisfactory colposcopy" with different degree of SCJ visibility. The term "unsatisfactory colposcopy" was abandoned because it has the connotation of an inadequate examination that needs to be repeated. ${ }^{4}$

Recently, an investigation published by our study group evaluated the risk of developing CIN2+ in consecutive 212 women diagnosed with high-risk HPV types with negative cytology results. We observed that HPV persistence correlated with both LSIL/CIN1 $+(P<.001)$ and $\mathrm{CIN} 2+(P<.001)$ in univariate analyses. Moreover, a 6-month increase in HPV persistence was associated with increased risk of developing $\mathrm{LSIL} / \mathrm{CIN} 1+(P=.010)$ and $\mathrm{CIN} 2+(P=.012)$ in multivariate analyses. ${ }^{10}$ The data reported in this previous investigation are in agreement with those reported in the present study.

Although several studies investigated the rate of occult CIN2+ diagnosed in women with high-risk cytology and unsatisfactory colposcopy, ${ }^{11-14}$ the present study is the first investigating the risk of developing CIN2+ over time in women for whom the trigger pap smear indicated the presence of lowgrade abnormalities. Our data will be useful during patients' counseling. Obviously, our findings have to be confirmed by larger prospective investigations.

Interestingly, our study suggested that there was no difference between HPV 16 to HPV 18 versus other HR-HPV types in predicting the risk of developing CIN $2+$. We observed that in a 10-year follow-up, there was no difference between different HPV types involved. This finding is in contrast with other results published in the literature. ${ }^{15}$ In fact, accumulating evidence suggested that HPV 16 and HPV 18 correlate with a greater risk of developing cervical dysplasia in comparison with other HPV types. ${ }^{15}$ However, the relative small sample size of the 2 subgroups of patients (HPV 16-HPV 18 vs other HR-HPV types) might influence this finding.

The inherent biases of the single-center retrospective study design are the main weaknesses of the present study. The main strength of the present investigation is represented by the potential clinical implication of our results. In fact, our data might be useful for patients' counseling and scheduling their follow-up. Four points of the present investigation deserve to be addressed: (1) we observed the patients diagnosed with a low-risk cervical cytology experienced a relative high risk of developing CIN2+, in case of HR-HPV persistence. However, owing to the small sample size and the relative low power of the study, these data have to be confirmed in other large investigations. (2) Our experience might not reflect the common practice since our study population is a selected group of patients referred into a National Cancer Center, instead of territorial centers. (3) In our analysis, type-specific HPV types are not associated with the risk of developing CIN2+. Other experiences suggested that type-specific HPV type(s) correlates with patient outcomes. ${ }^{8,13,14}$ (4) Smoking history might impact on patient outcomes. In the present study, tobacco use was defined as a dichotomous variable (yes vs no). We might speculate that magnitude of tobacco use as well as length of smoking might influence how this variable impact on our results.

In conclusion, the present study reported outcomes of women who had unsatisfactory colposcopy after the diagnosis of low-grade cervical cytological abnormalities. Our analysis suggested that HR-HPV persistence is the main factor predicting for the occurrence of CIN2+. In case of HR-HPV persistence, the risk of developing CIN2+ is not negligible. Further prospective studies are warranted in order to improve treatments of women with unsatisfactory colposcopy.

\section{Authors' Note}

Giorgio Bogani and Claudia Lombardo contributed to study design, data analysis, and manuscript writing. Francesca Taverna, Domenica Lorusso, and Francesco Raspagliesi contributed to study design, study planning, and manuscript writing. Antonino Ditto, Fabio Martinelli, and Mauro Signorelli contributed to study design, study conduct, and manuscript writing. Valentina Chiappa and Umberto Leone Roberti Maggiore contributed to data analysis and manuscript writing. Lavinia Mosca and Ilaria Sabatucci contributed to data collection and manuscript writing. Cono Scaffa contributed to data collection, study conduct, and manuscript writing.

\section{Declaration of Conflicting Interests}

The author(s) declared no potential conflicts of interest with respect to the research, authorship, and/or publication of this article.

\section{Funding}

The author(s) received no financial support for the research, authorship, and/or publication of this article.

\section{ORCID iD}

Fabio Martinelli, MD (D) http://orcid.org/0000-0002-4863-1747

\section{References}

1. Satterwhite CL, Torrone E, Meites E, et al. Sexually transmitted infections among US women and men: prevalence and incidence estimates, 2008. Sex Transm Dis. 2013;40(3):187-193.

2. Chesson HW, Dunne EF, Hariri S, Markowitz LE. The estimated lifetime probability of acquiring human papillomavirus in the United States. Sex Transm Dis. 2014;41(11):660-664.

3. Bogani G, Serati M, Cromi A, et al. Local anesthetic versus forced coughing at colposcopic-guided biopsy: a prospective study. Eur J Obstet Gynecol Reprod Biol. 2014;181:15-19. doi:10.1016/ j.ejogrb.2014.07.022.

4. Bornstein J, Bentley J, Bösze P, et al. 2011 colposcopic terminology of the International Federation for Cervical Pathology and Colposcopy. Obstet Gynecol. 2012;120(1):166-172. 
5. Makkar B, Batra S, Gandhi G, Zutshi V, Goswami D. Vaginal misoprostol versus vaginal estradiol in overcoming unsatisfactory colposcopy. Gynecol Obstet Invest. 2014;77(3):176-179. doi:10.1159/000358391.

6. Beniwal S, Makkar B, Batra S, Gandhi G, Goswami D, Zutshi V. Comparison of vaginal versus oral estradiol administration in improving the visualization of transformation zone (TZ) during colposcopy. J Clin Diagn Res. 2016;10(7):18-21.

7. International Agency for Research on Cancer, World Health Organization. Cervix Cancer Screening/IARC Handbooks of Cancer Prevention. Vol 10. Lyon, France: International Agency for Research on Cancer; 2005.

8. Bogani G, Martinelli F, Ditto A, et al. Human papillomavirus (HPV) persistence and HPV 31 predict the risk of recurrence in high-grade vaginal intraepithelial neoplasia. Eur J Obstet Gynecol Reprod Biol. 2017;210:157-165. doi:10.1016/j.ejogrb. 2016.12.020.

9. Koshiol J, Lindsay L, Pimenta JM, Poole C, Jenkins D, Smith JS. Persistent human papillomavirus infection and cervical neoplasia: a systematic review and meta-analysis. Am J Epidemiol. 2008; 168(2):123-137

10. Bogani G, Taverna F, Lombardo C, et al. Retrospective study of the influence of HPV persistence on outcomes among women with high-risk HPV infections and negative cytology. Int $J$ Gynaecol Obstet. 2017;138(1):62-68.

11. Veiga FR, Russomano FB, Camargo MJ, Monteiro AC, Tristão A, Silva GV. Prevalence of high-grade squamous intraepithelial lesions and cervical cancer among patients with unsatisfactory colposcopic examination, without visible lesion. Sao Paulo Med J. 2009;127(5):266-269.

12. El-Nashar SA, Shazly SA, Hopkins MR, Bakkum-Gomez JN, Famuyide AO. Loop electrosurgical excision procedure instead of cold-knife conization for cervical intraepithelial neoplasia in women with unsatisfactory colposcopic examinations: a systematic review and meta-analysis. J Low Genit Tract Dis. 2017;21(2):129-136.

13. Bogani G, Chiappa V, Martinelli F, Raspagliesi F. Type-specific HPV infection correlates with risk of recurrence of vulvar intraepithelial neoplasia usual type. Int J Cancer. 2017;140(7):1702. doi:10.1002/ijc.30585.

14. Bogani G, Martinelli F, Ditto A, et al. The association of pretreatment HPV subtypes with recurrence of VIN. Eur J Obstet Gynecol Reprod Biol. 2017;211:37-41.

15. Ciapponi A, Bardach A, Glujovsky D, Gibbons L, Picconi MA. Type-specific HPV prevalence in cervical cancer and high-grade lesions in Latin America and the Caribbean: systematic review and meta-analysis. PLoS One. 2011;6(10):e25493 